\title{
In peril from a perfect pathogen
}

It emerges that a fungal infection killing salamanders has many potential reservoirs and environmentally resistant spores can transmit disease. Urgent interventions are needed to save susceptible populations from extinction. SEE LETTER p. xxx

\section{Matthew C. Fisher}

Emerging infections caused by fungi are contributing to biodiversity loss ${ }^{1}$. Although how many fungal species inhabit the Earth is not known, estimates ${ }^{2}$ range from between 1.5 million to 5 million, and it seems reasonable to assume there is a deep pool of pathogenic fungi with previously undiscovered epidemiological traits. Accidental introductions of fungi to non-native habitats are an unfortunate byproduct of the intensification of global trade in forestry, agriculture and wildlife species. Through trade, a group of fungi known as the amphibian-parasitizing chytrids, have invaded ecosystems worldwide ${ }^{3}$. On page XXX, Stegen et al. $^{4}$ present an analysis of factors affecting the pathogenicity and transmission of one such fungus, Batrachochytrium salamandrivorans, which can kill salamander populations.

When a previously unknown fungus is identified as the cause of a disease outbreak, urgent research is required into the pathogen's biology to understand the disease-causing mechanisms and to determine how to control the organism. In the science-fiction film Alien, the heroine Ellen Ripley combats a 'perfect pathogen' that combines an unholy trinity of epidemiological features that lead to local extinction of its hosts: high virulence, no host immunity against the pathogen and an environmentally resistant transmission stage. One previously unknown 'alien' fungus now named $B$. salamandrivorans $(B s a l)^{5}$ invaded northern Europe from $A \mathrm{sia}^{6}$ around 2010 with extraordinarily high virulence in infected populations of fire salamanders (Salamandra salamandra), killing over $96 \%$ of these animals in the first recorded outbreak site. Stegen and colleagues present a fine-grain study uncovering a suite of epidemiological traits that give Bsal the characteristic of a highly successful 'perfect pathogen' during its invasion of the Robertville forests in Belgium, 57 kilometres from the initial outbreak site in the Netherlands.

Following the first sightings of dying fire salamanders in Robertville in 2014, the authors continuously monitored these amphibians as the fungal infection spread through the population. Their observations make for grim reading. The salamanders probability of becoming infected across 10 -day monitoring intervals was $33 \%$ and these animals had only a $13 \%$ chance of surviving longer than 10 days. The infection disproportionately infected sexually mature animals as they contact each other through social interactions, and their total population collapsed by over $90 \%$ within 6 months of the arrival of the infection. Two years later, less than $1 \%$ of the fire salamanders had escaped infection to roam their empty forest.

The authors made many key epidemiological observations that help to explain the ferocity of this outbreak. A series of infection trials revealed that $B s a l$ recovered from salamanders two years after the onset of the outbreak was just as lethal as the initial isolate and showed 
no signs of evolving towards a lesser virulence. Cycles of experimental inoculation of fire salamanders with $B s a l$ followed by subsequent treatment with antifungal drugs were used to test whether a protective immune response could be generated in the salamanders, but this was to no avail. The experimental animals all died, and it seems reasonable to infer that wild animals that escaped the initial outbreak have no immune defence against the pathogen. A study ${ }^{7}$ found that the expression of immune-system genes increased when Tylototriton wenxianensis salamanders were infected by Bsal's relative Batrachochytrium dendrobatidis, whereas infection of these salamanders by Bsal did not increase the expression of immune-system genes in the animals. This suggests that Bsal has evolved host immune-dampening properties.

However, the high virulence and lack of a salamander immune response alone would not account for the severity of this outbreak, and therefore Stegen and colleagues searched for factors that might enable high rates of fungal transmission. They looked for reservoirs of infection by analysing the forest soil for DNA traces of the presence of Bsal, which they found. This suggested that the fungus might be able to survive and remain infectious for lengthy periods outside of its infected host, a hypothesis that was confirmed by showing that infection could be transmitted to uninfected salamanders through contact with soil that had been contaminated by an infected fire salamander. Importantly, the authors demonstrated that environmental persistence was explained by the presence of a previously unknown type of resilient and non-motile thick-walled Bsal spore that has unique characteristics which promote its survival. These fungal spores are protected by a thickwalled water-repelling cell wall (an encysted spore) that can float at the interface between air and water rather than being actively swimming as occurs in another type of Bsal spore, known as a motile zoospore. The authors found that Bsal encysted spores had a substantially higher survival rate than the Bsal motile zoospores when exposed to pond micropredators, showing that the encysted form extends the infectious lifetime of the fungus.

Stegen et al. also searched for non-salamander reservoirs of infection that could be intensifying the outbreak. Although previous experiments ${ }^{6}$ suggested that the host-range of $\mathrm{Bsal}$ was limited to species of newts and salamanders, the authors found that a species of frog, Alytes obstetricans, could be infected by the fungus and transmit the disease to salamanders. Infection of alpine newts (Ichthyosaura alpestris) that share the forests with the salamanders alongside $A$. obstetricans then showed that the newts could develop chronic Bsal infections. That the alpine newts also show little evidence of protective immunity being mounted against re-infection by the pathogen indicates that they too are natural reservoirs of the pathogen.

So what are we to make of these findings? As the authors of the study state, the combination of multiple susceptible hosts alongside a virulent and environmentallypersistent pathogen appears to create a 'perfect storm' of infection that has so far led to the almost total destruction of fire salamanders in the populations infected to date. So far, the evidence appears to support the view that Bsal's dynamics are consistent with infectious spores building-up in multiple reservoirs across the timescale of an outbreak, 
creating a highly infected ecosystem. The authors tested whether the encysted spores could adhere to the feet of waterfowl and found this was the case. This might mean that birds can disperse $B s a l$ into previously uninfected and distant populations, perhaps explaining the extraordinarily rapid spread of the infection.

More must be done to try conserve not only fire salamanders, but also other susceptible amphibian species that have restricted ranges and are under direct threat of extinction from Bsal. It should become more of a priority to try to prevent introductions of other unknown pathogenic chytrids because, despite decades of research, no effective method has emerged yet to reduce their effect in the field ${ }^{8}$. It is unclear how Bsal can be combated in the wild beyond establishing 'amphibian arks' to safeguard susceptible species as the infection marches relentlessly onwards. Clearly, scientists, policy-makers and citizens have much to do if we are to help Europe's salamanders weather this 'perfect storm'.

Matthew C. Fisher is at the Department of Infectious Disease Epidemiology, Imperial College London, London W2 1PG, UK.

e-mail: matthew.fisher@imperial.ac.uk

1 Fisher, M. C., Gow, N. A. R. \& Gurr, S. J. Tackling emerging fungal threats to animal health, food security and ecosystem resilience. Philos TR Soc $B$ 371, doi:ARTN 2016033210.1098/rstb.2016.0332 (2016).

2 Blackwell, M. The Fungi: 1, 2, 3 ... 5.1 Million Species? Am J Bot 98, 426-438, doi:10.3732/ajb.1000298 (2011).

3 Fisher, M. C. et al. Emerging fungal threats to animal, plant and ecosystem health. Nature 484, 186-194, doi:Doi 10.1038/Nature10947 (2012).

$4 \quad$ Stegen et al., Nature22059 (2017).

$5 \quad$ Martel, A. et al. Batrachochytrium salamandrivorans sp nov causes lethal chytridiomycosis in amphibians. P Natl Acad Sci USA 110, 15325-15329, doi:Doi 10.1073/Pnas.1307356110 (2013).

6 Martel, A. et al. Recent introduction of a chytrid fungus endangers Western Palearctic salamanders. Science 346, 630-631, doi:Doi 10.1126/Science.1258268 (2014).

7 Farrer, R. A. et al. Genomic innovations linked to infection strategies across emerging pathogenic chytrid fungi. Nature Communications, 10.1038/ncomms 14742 (2017).[ref updated from in press. OK?]

8 Garner, T. W. J. et al. Mitigating amphibian chytridiomycoses in nature. Philos TR Soc B 371, doi:ARTN 2016020710.1098/rstb.2016.0207 (2016).

Figure 1 | Salamanders under threat from a fungal pathogen. Stegen et $a .^{4}$ investigated factors that might be contributing to the rapid spread of a Batrachochytrium salamandrivorans (Bsal) fungal infection in salamander (Salamander salamander) populations in Europe. The authors find that other species including Alytes obstetricans frogs (known as midwife toads) and Ichthyosaura alpestris newts can act as fungal reservoirs and therefore might be able to transmit infection to salamanders. Infected salamanders can 
spread the disease as fungal spores disperse from their bodies and the authors discovered a previously unknown type of $B s a l$ spore, termed an encysted spore, that is more resistant to predation by zooplankton than the motile aquatic form of $\mathrm{Bsal}$ spores. The authors found that the encysted spores can adhere to bird feet and speculate that birds might have a role in pathogen dispersal.

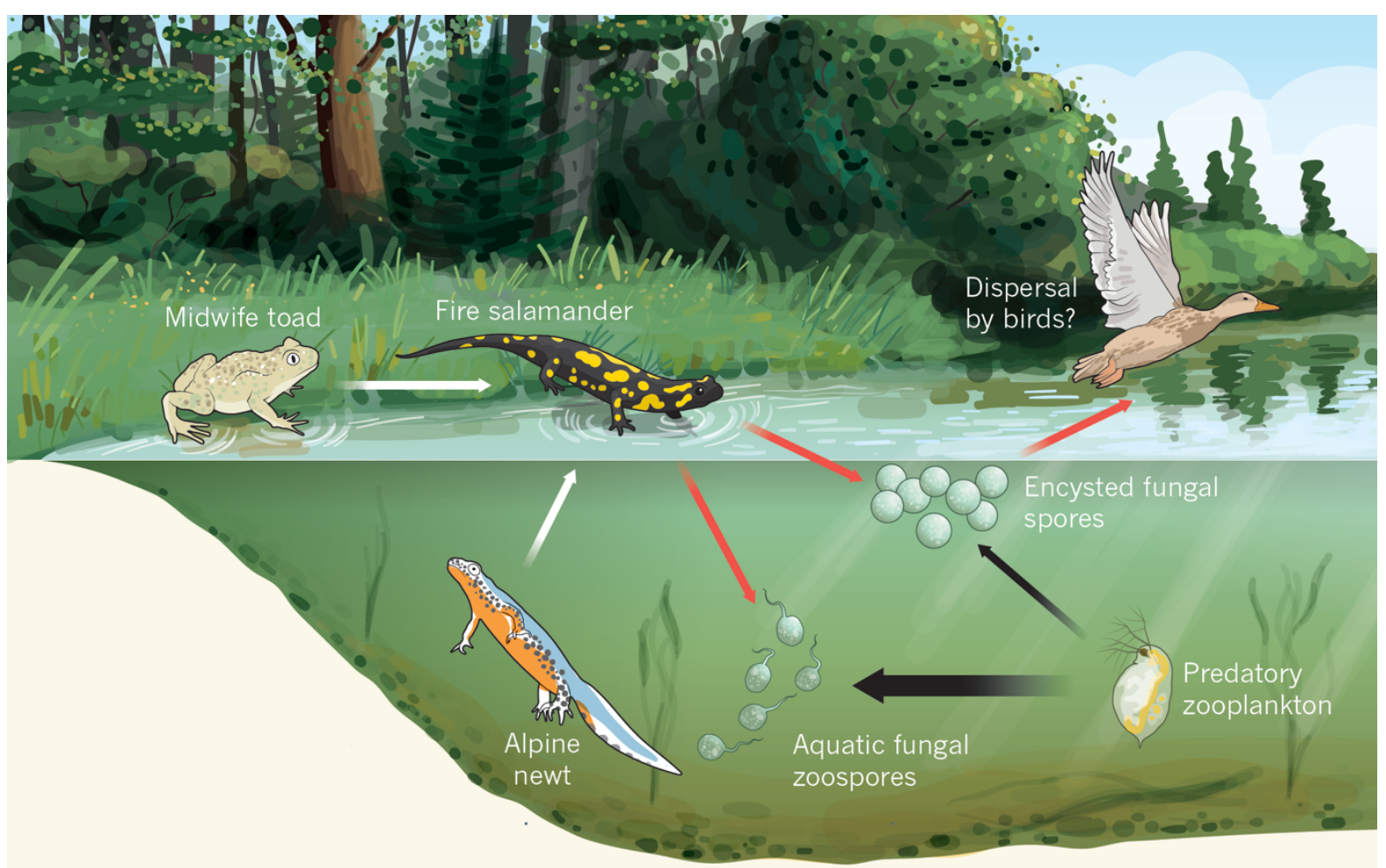

